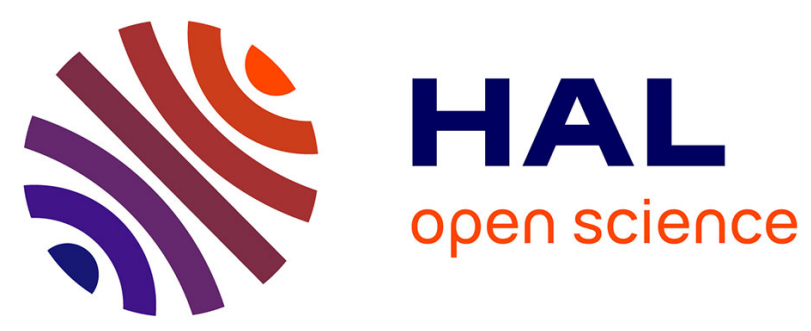

\title{
Does traffic-related calibration of car-following models provide accurate estimations of vehicle emissions?
}

Thamara Vieira da Rocha, Ludovic Leclercq, Marcello Montanino, Céline Parzani, Vicenzo Punzo, Biagio Ciuffo, Daniel Villegas

\section{- To cite this version:}

Thamara Vieira da Rocha, Ludovic Leclercq, Marcello Montanino, Céline Parzani, Vicenzo Punzo, et al.. Does traffic-related calibration of car-following models provide accurate estimations of vehicle emissions?. Transportation research part D: Transport and Environment, 2015, 34, pp. 267-280. 10.1016/j.trd.2014.11.006 . hal-01215763v3

\section{HAL Id: hal-01215763 \\ https://hal.science/hal-01215763v3}

Submitted on 28 Jan 2016

HAL is a multi-disciplinary open access archive for the deposit and dissemination of scientific research documents, whether they are published or not. The documents may come from teaching and research institutions in France or abroad, or from public or private research centers.
L'archive ouverte pluridisciplinaire HAL, est destinée au dépôt et à la diffusion de documents scientifiques de niveau recherche, publiés ou non, émanant des établissements d'enseignement et de recherche français ou étrangers, des laboratoires publics ou privés. 
Transportation research part D: Transport and Environment, vol. 34, pp. 267-280, DOI: 10.1016/j.trd.2014.11.006

February 23, 2014

\title{
Does traffic-related calibration of car-following models provide accurate estimations of vehicle emissions?
}

\author{
Thamara Vieira da Rocha ${ }^{\mathrm{a}}$, Ludovic Leclercq ${ }^{\mathrm{a}, 1}$, Marcello Montanino ${ }^{\mathrm{b}}$, Céline Parzani ${ }^{\mathrm{a}}$, \\ Vincenzo Punzo ${ }^{\mathrm{b}}$, Biagio Ciuffo $^{\mathrm{c}}$, Daniel Villegas ${ }^{\mathrm{a}}$
}

(a)

Université de Lyon, Lyon, France ; IFSTTAR, LICIT, Bron, France ; ENTPE, LICIT, Vaulx-en-Velin, France

(b)

Department of Transportation Engineering, Università di Napoli Federico II, Italy

(c)

Institute for the Energy and Transport, European Commission - Joint Research Centre

Paper $n^{\circ} 14-\mathrm{xxxx}$

Paper submitted for publication to Transportation Research part D

Original submission date: 23/02/2014

\footnotetext{
${ }^{1}$ Corresponding author - ludovic.leclercq@ifsttar.fr. Tel +33 472047716.
} 


\begin{abstract}
A convenient way to assess the traffic fuel consumption or pollutant emissions is to couple a microscopic traffic flow model with an instantaneous emission model. Traffic models are usually calibrated using goodness of fit indicators that are related to the traffic behavior. Thus, this paper aims to investigate how such a calibration influences the accuracy of fuel consumption and NOx and PM estimations. Two traffic models are investigated: Newell's and Gipps' models. It appears that Gipps' model provides the closest simulated trajectories when compared to real ones. Interestingly, a reverse ranking is observed for fuel consumption, NOx and PM emissions. For both models, the vehicle emissions of a single vehicle are very sensitive to the calibration. This is confirmed by a global sensitivity analysis of the Gipps model that shows that non-optimal parameters significantly increase the variance of the outputs. Fortunately, this is no longer the case when emissions are calculated for a group of many vehicles. Indeed, the mean errors for platoons are close to $10 \%$ for the Gipps model and always lower than $4 \%$ for the Newell's model. This latter model then clearly outperforms the first one for emission calculations. Another interesting property is that optimal parameters for each vehicle can be replaced by the mean values with no discrepancy for the Newell model and low discrepancies for the Gipps model when calculating the different emission outputs. Finally, this study presents preliminary results that show that multi-objective calibration methods are certainly the best direction for future works on the Gipps model. Indeed, the accuracy of vehicle emissions can be highly improved with negligible counterparts on the traffic model accuracy.
\end{abstract}

Keywords: Microscopic traffic model, car-following, vehicle emissions, fuel consumption, calibration, sensitivity analysis 


\section{Introduction}

The assessment of the environmental impact of intelligent transportation systems is a key issue in the context of sustainable mobility. Thus most of the traffic micro-simulation models available on the market include tools for modelling exhaust emissions of vehicles and, in particular, instantaneous (modal) emission models. These models use high frequency measurements to map emissions at a time to their generating engine state, thus providing specific emission factors (also) for new, unmeasured driving cycles. Once such models are fed with real world driving cycles of a traffic flow, calculations are expected to capture the impact on emissions of those specific traffic conditions and control schemes. Unfortunately driving cycles of all the vehicles within a timespace domain are hardly ever available, e.g. the data collected in the NGSIM program, (Federal Highway Administration, 2006). Their intrinsic complexity, also due to the increasing impact of new technologies, e.g. time responses of the engine electronic management system to the lambda sensor, which considerably differ among vehicles, is the other major drawback of instantaneous modelling. However, the massive development of traffic flow micro-simulation, and hence the chance to simulate driving cycles of all the vehicles on a network, is promoting the adoption of such integrated dynamic traffic-emissions modelling.

Yet the problem with the use of micro-simulation to derive driving cycles is that the whole approach must be validated, e.g. (Int Panis et $a l, 2006)$, but at present, the accuracy of the trajectories drawn by the model remains unknown for such an application. Preliminary results (Vieira da Rocha et al, 2013) show that simplified driving cycles classically provided by traffic simulators introduce bias when calculating the fuel consumption. Fortunately, such errors remain relatively low for a given cycle and vanish when lots of cycles are gathered to determine the total fuel consumption. However, this study has not considered the specific influence of the traffic model: simplified driving cycles are directly derived from real measures by using filtering techniques. Furthermore, traffic models are generally calibrated against aggregate measurements and the objective of calibration is to improve the reproduction of aggregate traffic dynamics rather than individual driving cycles, e.g. (Ciuffo et al, 2008; Toledo et al, 2004). Thus, car-following models have generally underperformed in reproducing real world behaviors of individual vehicles, e.g. (Brockfeld et al, 2004; Punzo and Simonelli, 2005). The problem of reproducing trajectories for emission calculation is even more complex, since the way in which car-following models are calibrated (even when detailed trajectory data are used) may not be the most appropriate with a view to calculating traffic-related externalities. Note that because driving cycles correspond to the derivative of the trajectories, both terminologies are used indifferently in this paper. 
This work investigates the accuracy of results when atmospheric emissions and fuel consumption are calculated from simulated trajectories instead of the real ones. A set of 90 vehicle trajectories corresponding to nine different platoons gathered from the NGSIM database (Federal Highway Administration, 2006) is used as reference. These trajectories are filtered to remove measurements noise. Two car-following models are used to generate simulated trajectories from the leader one: Newell's (Newell, 2002) and Gipps' (Gipps, 1981) models. These two models are calibrated using standard goodness of fit indicators related to vehicle trajectories. Atmospheric emissions and fuel consumption are then calculated using the state-of-the-art PHEM (Passenger Car and Heavy Duty Emission Model) model (Hausberger et al, 2009; Luz et al, 2013). It is an instantaneous vehicle emission model developed by the TU Graz since 1999. In this paper, we will focus on fuel consumption, NOx and Particle Matters (PM) and investigate the accuracy of emission calculations at both individual (vehicle) and global (platoon) levels.

The paper is organized as follows: Section 2 provides some background about the two traffic models, their calibration using classical goodness of fit and the emission model. Section 3 presents the different sensitivity tests that have been carried out to assess the influence of the traffic model calibration on the accuracy of the emission calculations. Section 4 presents the results for the 90 vehicles and 9 platoons and investigates the connections between the traffic model parameters and the emission estimation. A global sensitivity analysis is also proposed. Finally, section 5 proposes a brief discussion. It notably shows that a multi-objective calibration method clearly improves the emission results while it only slightly deteriorates the accuracy of simulated trajectories.

\section{Background}

\subsection{Traffic data}

Vehicle trajectories come from the I-80 NGSIM dataset (Federal Highway Administration, 2006). Video recordings were performed on a $500 \mathrm{~m}$ length section of the interstate 80 in Emeryville (California, USA) during the peak hour (congested traffic conditions with speed always lower than $50 \mathrm{~km} / \mathrm{h}$ ). Nine platoons of 10 vehicles were extracted from the whole data with the following requirements: (i) no lane changings should happen within the platoon when crossing the whole section and (ii) the platoon should entail at least 10 vehicles. This guarantees that the interactions between a vehicle and its leader can be characterized by a car-following rule without any external distractions (Chiabaut et al, 2009). 
Vehicle positions were automatically extracted from the video recording with a frequency of $10 \mathrm{~Hz}$. This introduces measurement errors that may lead to significant bias when performing the calibration of car-following models, e.g. (Ossen and Hoogendoorn, 2008; Punzo et al, 2011). The bias are even greater when differential variables, like speed or acceleration are calculated. Thus, Treiber et al (2008) and Rakha et al (2001) have shown that using the raw data without filtering leads to arbitrarily high estimates for fuel consumption.

Trajectories data should then be filtered before performing (i) the car-following model calibration and (ii) the emission calculations. The following filtering process was applied to reduce the measurement noise. First, a filtered trajectory is defined as a succession of $n$ quadratic functions. Such functions continuously connect at $n-1$ time points named knots (Marczak and Buisson, 2012; Xin et al, 2008). Derivatives are also continuous at knots. Thus, the trajectories are approximated by a function with piecewise constant accelerations and continuous speed. These assumptions seem physically reasonable. Second, a genetic algorithm is applied to determine the optimal positions of knots for $n$ between 6 and 20. The objective function for this global optimization process is the root mean square error (RMSE) between filtered and original positions. Note that for a given set of knot positions, the parameters of the quadratic functions can simply be derived using a quadratic regression. A supplementary constraint is also introduced to avoid time intervals between two knots that lead to acceleration values lower than $-2 \mathrm{~m} / \mathrm{s}^{-2}$ or higher than $2 \mathrm{~m} / \mathrm{s}^{2}$. Third, $n$ values are tested in increasing order. The optimal $n$ value is considered reached when no significant RMSE improvements are observed compared to the previous step.
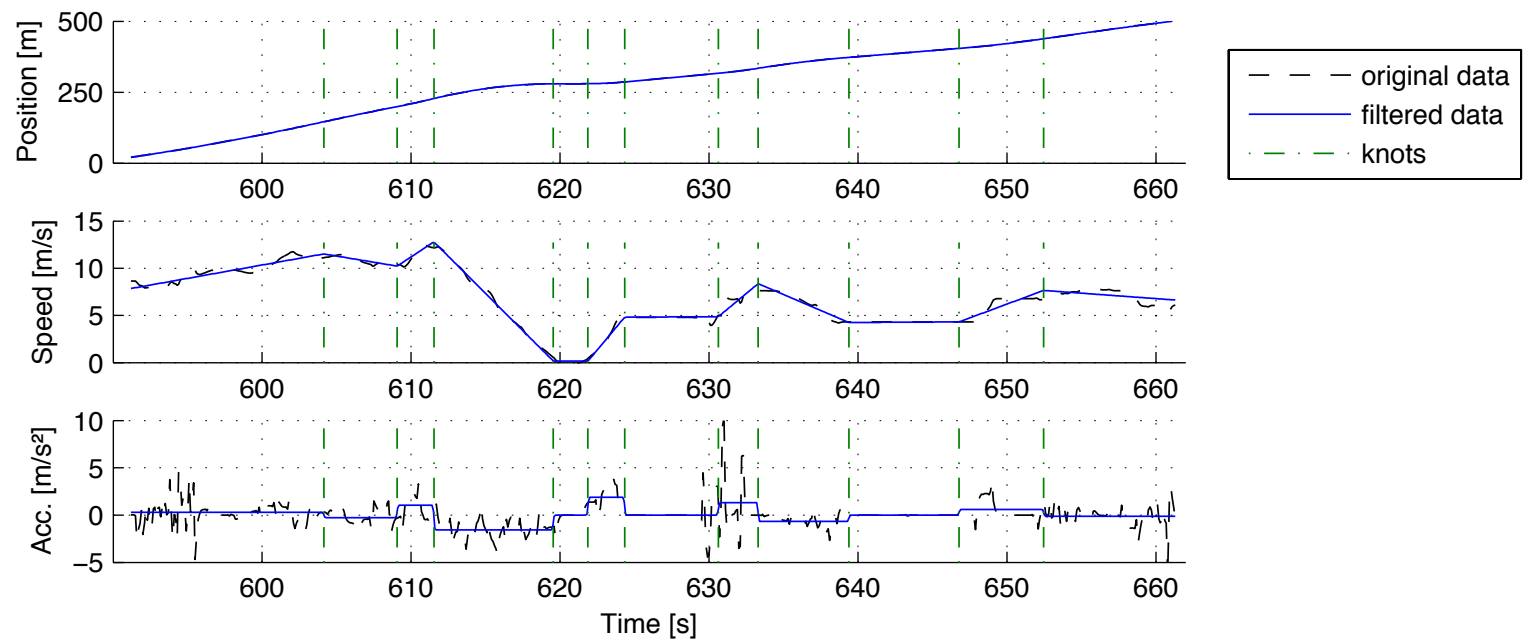

Fig. 1. Position, speed and acceleration profiles before and after filtering 
Fig. 1 presents the results of the filtering for a particular trajectory. The vertical lines on the figure correspond to the knot locations. The mean RSME for the 90 filtered trajectories is $0.3 \mathrm{~m}$. The individual RMSE varies from 0.1 to $0.8 \mathrm{~m} .80 \%$ of the filtered trajectories experiment RMSE between 0.2 and $0.6 \mathrm{~m}$.

\subsection{Calibration of Newell's and Gipps' car-following models}

The Newell model (Newell, 2002) is certainly the simplest car-following rule and only requires two parameters in congestion: the wave speed $w$ and the jam spacing $s$. The position $x_{j}(t+\Delta t)$ of vehicle $j$ at time $t+\Delta t$ can be derived from its position $x_{j}(t)$ and that of its leader $x_{j-1}(t)$ at time $t$ :

$$
x_{j}(t+\Delta t)=\left(1-\alpha_{j}\right) x_{j}(t)+\alpha_{j} x_{j-1}(t)-w_{j} \Delta t \text { with } \alpha_{j}=w_{j} s_{j} \Delta t \leq 1
$$

Newell's model has been proven (Leclercq et al, 2007) to be equivalent to the macroscopic LWR model (Lighthill \& Whitham, 1955; Richards, 1956). The calibration of the Newell model is performed for each vehicle pair following the methodology described in Chiabaut et al (2010) that will not be recapped here. This task provides the optimal values $w_{j}$ and $s_{j}$ for all vehicles.

The Gippsmodel (Gipps, 1981) is a safety-based model. The speed $v_{j}\left(t+\tau_{j}\right)$ of vehicle $j$ at time $t+\tau_{j}$ is derived from its leader speed and position according to the current driving modes: free-flow, see eq. (3) or congestion, see eq. (4). $\tau_{j}$ is the reaction time of driver $j$. Note that even if our data are always congested the Gipps model may sometimes switch to the free-flow mode for short time periods. Thus, both modes and the switching rule have to be considered:

$$
\begin{gathered}
v_{j}\left(t+\tau_{j}\right)=\min \left(v_{j}^{F}\left(t+\tau_{j}\right), v_{j}^{C}\left(t+\tau_{j}\right)\right) \\
v_{j}^{F}\left(t+\tau_{j}\right)=v_{j}(t)+2.5 a_{\max , j} \tau_{j}\left(1-\frac{v_{j}(t)}{v_{\max , j}}\right)\left(0.025+\frac{v_{j}(t)}{v_{\max , j}}\right) \\
v_{j}^{C}\left(t+\tau_{j}\right)=-d_{\text {max }, j}\left(\tau_{j}+\theta_{j}\right)+\sqrt{d_{\max , j}^{2}\left(\frac{\tau_{j}}{2}+\theta_{j}\right)^{2}+d_{\text {max }, j}\left(2\left(x_{j-1}(t)-s_{j-1}\right)-\tau_{j} v_{j}(t)+\frac{v_{j-1}^{2}(t)}{d_{\text {max }, j-1}}\right)}
\end{gathered}
$$

where:

$v_{j}(t)$ and $v_{j-1}(t)$ are respectively the speed of the following and leading vehicle at time $t$;

$a_{\max , j}, v_{\max , j}, d_{\max , j}$ are respectively the maximum acceleration, speed and deceleration for vehicle $j$ and $d_{\text {max }, j-1}$ is the maximum deceleration for vehicle $j-1$ as perceived by vehicle $j$;

$\tau_{j}$ is the apparent reaction time; 
$\theta$ is an additional comfort time lag that allows the follower not to brake always at his or her maximum desired rate, usually $\theta_{j}=\tau_{j} / 2$;

$s_{j-1}$ is the jam spacing.

For further details on the simulation setup and the initialization conditions adopted in this paper, please refer to Wilson (2001). The Gipps model calibration was performed for the following six parameters: $\tau, s_{j-1}, v_{\max , j}, a_{\max , j}$, $d_{\text {max }, j}, d_{\text {max }, j-1}$. This task consists in solving an optimization problem for each vehicle pair that minimizes the speed RMSE between the simulated and the original trajectories of the followers. All details are provided in (Ciuffo et al, 2012) and will not be recapped here.

(a)

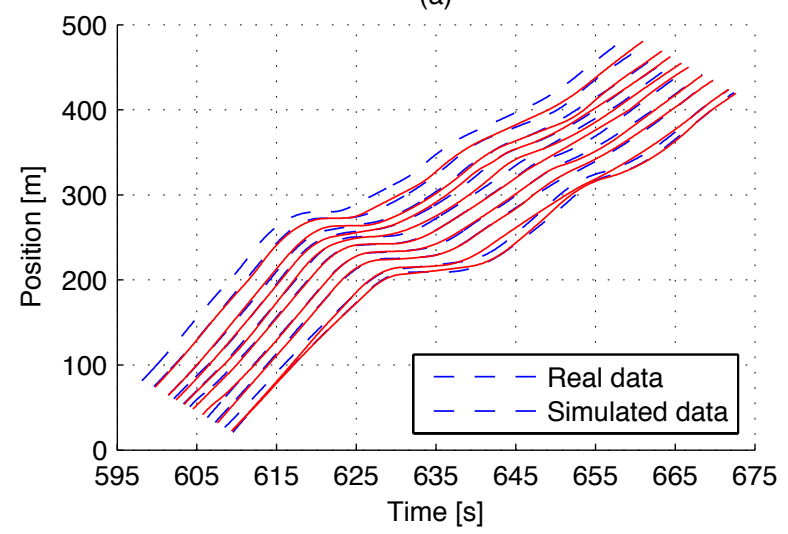

(b)

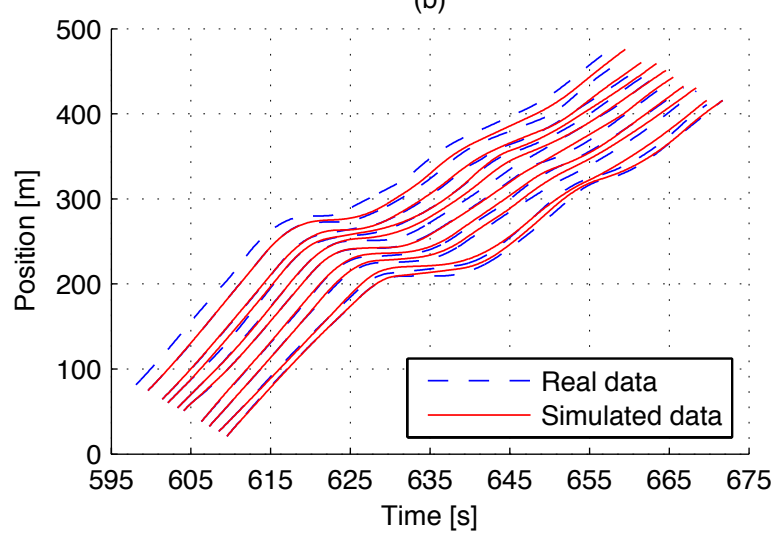

Fig. 2. Original and simulated trajectories using (a) Newell's model and (b) Gipps's model

Fig. 2 presents the real and simulated trajectories after calibration for a given platoon and both models. A visual inspection shows that the simulated trajectories with the optimal parameters are globally close to the experimental data for both models. A refined investigation consists in comparing the speed RMSE for both models and all vehicle pairs. Fig. 3 presents the resulting cumulative distribution for all pairs. It appears that the calibration of the Gipps model provides more accurate results than the calibration of the Newell model with respect to the driving cycle. The mean RMSE is equal to $0.5 \mathrm{~m} / \mathrm{s}$ for Gipps compared to $0.7 \mathrm{~m} / \mathrm{s}$ for Newell. This is not surprising because Gipps' model has 6 parameters while Newell's model has only two. Anyway, from a traffic point of view, these two models can be considered as accurate, because the RMSE $95^{\text {th }}$ percentile is never higher than $0.7 \mathrm{~m} / \mathrm{s}$ for Gipps and than $1.1 \mathrm{~m} / \mathrm{s}$ for Newell. 


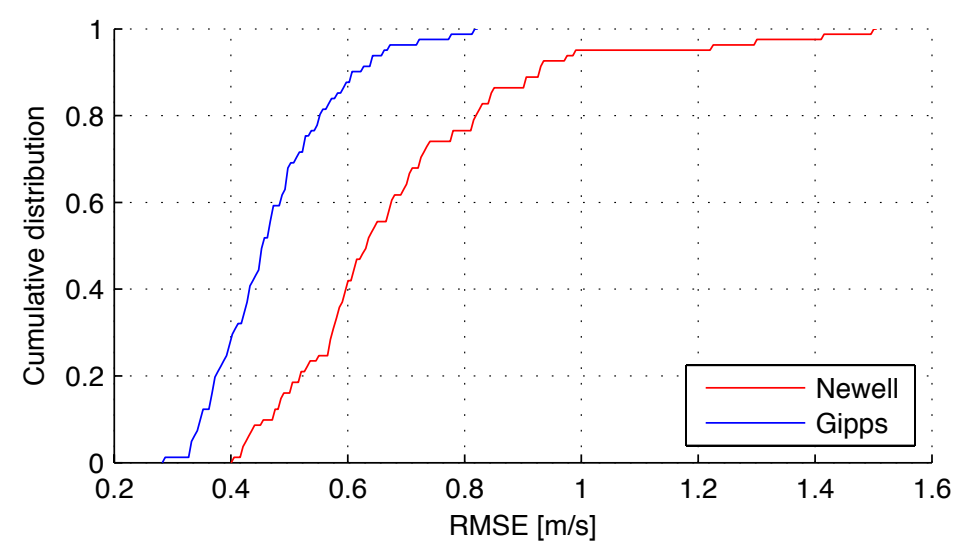

Fig. 3. RMSE cumulative distribution for all vehicle pairs

\subsection{Emission model: PHEM}

In this study, we use a well-established instantaneous emission model in Europe: the Passenger car and Heavy duty Emission Model (PHEM). This model has been developed by the Graz University of Technology since 1999, within the FP 7 project ARTEMIS, COST 346 and the HBEFA development. PHEM is based on an extensive European set of vehicle measurements and covers passenger cars, light duty vehicles and heavy duty vehicles from city buses up to 40 ton semi-trailers.

PHEM calculates the engine power in $1 \mathrm{~Hz}$ based on the given courses of vehicle speed and road gradient, the driving resistances and the losses in the transmission system. The $1 \mathrm{~Hz}$ course of engine speed is simulated based on the transmission ratios and a gear-shift model. The model results then are the $1 \mathrm{~Hz}$ courses of engine power, engine speed, fuel consumption and emissions of $\mathrm{CO}_{2}, \mathrm{CO}, \mathrm{HC}, \mathrm{NOx}, \mathrm{NO}$, particle mass (PM) and particle number (PN). The supporting data-set includes gasoline and diesel vehicles from EURO 0 to EURO 6 (Hausberger et al, 2009).

In this paper, we will restrict our investigation to passenger cars and the most common pollutants: $\mathrm{CO}_{2}$ (or equivalently fuel consumption), NOx and Particle Matters (PM). The fleet composition corresponds to the national projection for 2015 in France (CETU, 2012), see Tab. 1.

\begin{tabular}{llllllll}
\hline & Euro 0 & Euro 1 & Euro 2 & Euro 3 & Euro 4 & Euro 5 & Euro 6 \\
\hline Diesel & $0.2 \%$ & $0.7 \%$ & $2.4 \%$ & $12.9 \%$ & $34.1 \%$ & $49.7 \%$ & $0.0 \%$ \\
Gazole & $0.3 \%$ & $1.9 \%$ & $6.9 \%$ & $20.0 \%$ & 35.4 & 35.5 & $0.0 \%$ \\
\hline
\end{tabular}

Tab. 1: Passenger car fleet scenario for 2015 in France: $86 \%$ of diesel car (CETU, 2012) 


\section{Method}

Different tests will be undertaken to assess the impact of the car-following parameters on the vehicle emissions. The first group of tests focuses on the car-following process at a local (vehicle pair) and a global (platoon) level. The second study corresponds to a global sensitivity analysis based on the classical method firstly introduced by Sobol (1990).

\subsection{Different tests to investigate the influence of car-following laws}

Let denote $T$ the generic function that provides the follower trajectory from the leader one. $T$ may correspond either to the Newell or to the Gipps model. The calibration task provides the optimal set of parameters $C_{j}$ for each vehicle $j$ of all platoons. Let us remember that the number of parameters is 6 for Gipps and 2 for Newell and that the leader of $j$ is $j-1$.

Test 1 and test 2 compare the fuel consumption, the NOx and PM emissions associated to the real and the simulated trajectory of vehicle $j$ for both traffic models. Optimal parameters are used for all vehicles. The only difference is that test 2 propagates the errors within the platoon by using the simulated trajectory for vehicle $j-1$ instead of the real trajectory like in test 1.

\begin{tabular}{cl}
\hline Test & \multicolumn{1}{c}{ Description } \\
\hline Test & $\begin{array}{l}\text { The simulated trajectory } S_{j} \text { is calculated from the leader real trajectory } X_{j-1} \text { and the optimal set } C_{j} \text { of parameters } \\
\mathbf{1}\end{array}$ \\
associated to $j: S_{j}=T\left(X_{j-1}, C_{j}\right)$ \\
\hline Test & $S_{j}$ is calculated from the leader simulated trajectory $S_{j-1}$ and $C_{j}: S_{j}=T\left(S_{j-1}, C_{j}\right)$ \\
$\mathbf{2}$ & \\
\hline Test & $S_{j}$ is calculated from the leader simulated trajectory $S_{j-1}$ and the set $C$ of parameters defined by the mean value of the \\
$\mathbf{3}$ & optimal parameters for all the vehicles: $S_{j}=T\left(S_{j-1}, C\right)$ \\
\hline
\end{tabular}

Tab. 2: Definition of the different tests about the influence of car-following model calibration

Test 3 investigates how non-optimal parameters worsen the estimation of the emissions. Indeed, in practice when using a microscopic traffic simulator, the optimal parameters for each particular vehicle are unknown. At best, parameters are drawn from a calibrated distribution and assigned to the generated vehicles. At least, only the mean value of the distribution is available. Thus, in test 3 , the optimal parameters are replaced by the mean value of the parameters for all the available vehicles. Note that test 3 is only performed for the Gipps model. Indeed, for the Newell model the follower trajectory corresponds to the leader one translated by a vector that depends on the car-following parameters. Such parameters influence the traffic wave propagation but not the simulated speed profile of the follower. Thus, the emissions are the same for a vehicle $j$ whatever the Newell model's parameters are. The results for test 3 will be the same as for test 2 for the Newell model as far as the emission calculations are concerned. All the tests are recapped in Tab. 2. 


\subsection{Global sensitivity analysis}

Another way to study the influence of the car-following parameters on the estimation of vehicle emissions is to perform a global sensitivity analysis. This method was firstly introduced by Sobol (1990) and consists in quantifying the contribution of each input parameters to the output variances. A full description of the method can be found in (Saltelli et al, 2008) or (Punzo and Ciuffo, 2011). Here, the outputs are the difference of the fuel consumption, the level of NOx and PM emissions between the real and the simulated trajectory for vehicle $j$. We have restricted the global sensitivity analysis to the Gipps model because the Newell model has too few parameters. Thus, the considered inputs are the Gipps parameters with lower and upper bounds defined in Tab. 3 . We have introduced a last input that corresponds to the vehicle number $j$. Indeed, because the optimal parameters for a specific vehicle are not accessible in practice, we have decided to assess the influence of the parameters for a randomly drawn vehicle. In practice, this is the case when a traffic simulator affects to a vehicle a set of parameters drawn from the correlated distributions of the calibrated ones. The simple way to implement this option is to incorporate the vehicle identification as an input for the global sensitivity analysis.

Two indicators will be used to analyze the results. The first is the first order sensitivity index $S_{i}$ for each parameter $i$ and a given output. This indicator represents the ratio between the variance of this output when parameter $i$ is fixed and the total variance. It describes the direct contribution of parameter $i$ to the total variance. Note that the sum of $S_{i}$ should be equal to 1 . A low $S_{i}$ means that the parameter $i$ weakly influences the considered output. The second indicator is the total effect index $S T_{i}$. This indicator jointly characterizes the direct contribution of parameter $i$ and its combined contribution with all the other parameters (second order interactions). Low $S_{i}$ but high $S T_{i}$ reveals that parameter $i$ has a low contribution by itself but is more highly involved in joined contributions with other parameters.

\begin{tabular}{ccc}
\hline Parameters & Lower bound & Upper bound \\
\hline$\tau_{j}[\mathrm{~s}]$ & 0.1 & 3.0 \\
$v_{\max , j}[\mathrm{~m} / \mathrm{s}]$ & 12,0 & 50.0 \\
$a_{\max , j}\left[\mathrm{~m} / \mathrm{s}^{2}\right]$ & 0.4 & 8.0 \\
$d_{\text {max }, j}\left(\mathrm{~m} / \mathrm{s}^{2}\right)$ and $d_{\text {max }, j-1}\left[\mathrm{~m} / \mathrm{s}^{2}\right]$ & 0.4 & 10.0 \\
$s_{j}[\mathrm{~m}]$ & 4 & 10 \\
$j$ (first vehicle of each platoon are disregarded) & 2 & 90 \\
\hline
\end{tabular}

Tab. 3: Lower and upper bounds for the global sensitivity analysis 


\section{Results}

The local error $\varepsilon[\%]$ is defined as the relative difference in emissions between real and simulated trajectories for each individual follower. The global error $E[\%]$ corresponds to the relative difference in emissions for all the vehicles of a platoon except for the first leader. Local and global errors are calculated for fuel consumption (FC), NOx and PM emissions. The speed RMSE between real and simulated trajectories is also monitored for analysis.

\subsection{Test 1}

Test 1 only focuses on the influence of the car-following law at a local level (vehicle pairs). The results of the calibration from a traffic point of view has been presented in section 2.2, see Fig. 3. Both models appear accurate while Gipps provides the lowest speed RMSE at the cost of four extra parameters. The cumulative distributions of $\varepsilon$ and $E$ for the three considered emission outputs are presented in Fig. 4. For each distribution, the mean value $m$, the standard deviation $\sigma$, the $5^{\text {th }}$ and the $95^{\text {th }}$ percentiles $\left(q_{5 \%}\right.$ and $\left.q_{95 \%}\right)$ are given in Tab. 4 . This table also provides for the same statistics the absolute values of the real and the simulated emissions.
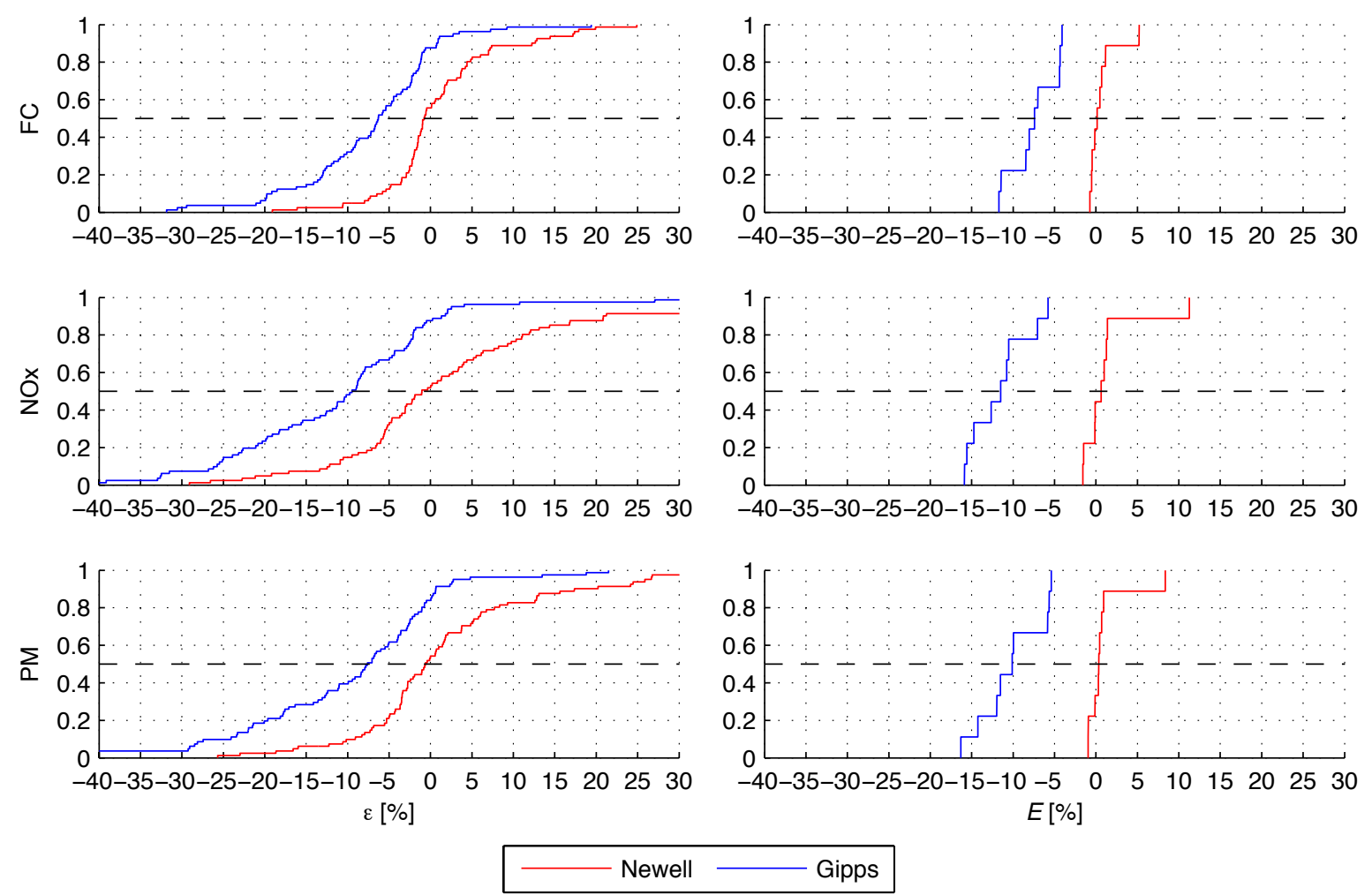

\section{Fig. 4. Cumulative distribution for the local and global errors - Test 1}

The analysis of the local errors shows that the Newell model appears significantly more accurate than the Gipps model for all the outputs. Indeed, the mean $\varepsilon$ for fuel consumption (respectively NOx and PM emissions) is equal to $0.9 \%$ (respectively $2.6 \%$ and $1.7 \%$ ) for the Newell model and to $-7.3 \%$ (respectively $-11 \%$ and $-9.7 \%$ ) for the 
Gipps model. The individual local errors can be much larger with an $\varepsilon$ value for fuel consumption from $-9.1 \%$ to $17.3 \%$ for Newell and from $-20.7 \%$ to $3.1 \%$ for Gipps. Note that these interval bounds correspond to the $5^{\text {th }}$ and the $95^{\text {th }}$ percentiles and not to the minimum and maximum values. This notation will be applied in the whole sequel of the paper. The lengths of these intervals are even higher for the NOx and PM emissions: $58 \%$ and $32.4 \%$ for Newell and $35.8 \%$ and $32.9 \%$ for Gipps. Thus, we can conclude from this first observation that even with optimal parameters, both car-following models may lead to significant errors when estimating the fuel consumption or the NOx and the PM emissions of a single vehicle. Fortunately, the mean error is very low for Newell's model and not too high for the Gipps's model. That means that errors compensate when considering enough vehicles. This is confirmed in Fig. 4 when the global error is calculated. The mean $E$ for fuel consumption is equal to $0.6 \%$ for the Newell model $(-0.7<E<5.2 \%)$ and to $-7.4 \%$ for the Gipps model $(-11.7 \%<$ $E<-4.1 \%$ ). The same trends are observed for the NOx and PM global errors, see Tab. 4.

\begin{tabular}{cccccccccc}
\hline & & \multicolumn{3}{c}{ Newell's model } & \multicolumn{4}{c}{ Gipps's model } \\
& & $\boldsymbol{m}$ & $\boldsymbol{\sigma}$ & $\boldsymbol{q}_{\mathbf{5} \%}$ & $\boldsymbol{q}_{\mathbf{9 5} \%}$ & $\boldsymbol{m}$ & $\boldsymbol{\sigma}$ & $\boldsymbol{q}_{\mathbf{5} \%}$ & $\boldsymbol{q}_{\mathbf{9 5} \%}$ \\
\hline \multirow{4}{*}{ FC } & Real [g] & 36,8 & 8,3 & 27,4 & 51,7 & 35,8 & 8,1 & 26,8 & 49,6 \\
& Sim. [g] & 37,0 & 8,2 & 28,2 & 50,9 & 33,1 & 7,8 & 24,3 & 47,9 \\
& $\boldsymbol{\varepsilon}[\%]$ & 0,9 & 7,3 & $-9,1$ & 17,3 & $-7,3$ & 8,4 & $-20,7$ & 3,1 \\
& $E[\%]$ & 0,6 & 1,8 & $-0,7$ & 5,2 & $-7,4$ & 2,9 & $-11,7$ & $-4,1$ \\
\hline \multirow{4}{*}{ NOx } & Real [mg] & 304 & 86 & 201 & 478 & 295 & 82 & 198 & 448 \\
& Sim. [mg] & 308 & 84 & 212 & 472 & 262 & 79 & 174 & 418 \\
& $\boldsymbol{\varepsilon}[\%]$ & 2,6 & 15,6 & $-20,1$ & 37,9 & $-11,0$ & 12,8 & $-32,5$ & 3,2 \\
& $E[\%]$ & 1,4 & 3,9 & $-1,6$ & 11,3 & $-11,6$ & 3,6 & $-15,9$ & $-5,8$ \\
\hline \multirow{4}{*}{ PM } & Real [mg] & 12,5 & 2,9 & 9,0 & 18,1 & 12,2 & 2,8 & 8,9 & 17,6 \\
& Sim. [mg] & 12,6 & 2,9 & 9,2 & 17,7 & 10,9 & 2,7 & 7,8 & 16,5 \\
& $\boldsymbol{\varepsilon}[\%]$ & 1,7 & 12,0 & $-16,2$ & 26,2 & $-9,7$ & 11,9 & $-29,2$ & 3,7 \\
& $E[\%]$ & 1,0 & 2,8 & $-0,9$ & 8,4 & $-10,1$ & 3,9 & $-16,3$ & $-5,4$ \\
\hline
\end{tabular}

Tab. 4: Emission results for Test 1

The results from this first test are quite surprising because the Gipps model outperforms the Newell model from a traffic point of view but clearly underperforms when vehicle emissions are considered. The most immediate explanation is that the speed profiles are more smoothed when calculated with the Gipps model. Indeed, Newell's model only translates the leader trajectory following the direction of traffic wave propagation. A detailed and synchronized analysis of the speed profiles and the evolution would be necessary to fully identify the reasons why Gipps' model underperforms, see (Vieira da Rocha et al, 2013 for the methodology. This is out of the scope of the present paper. Note that further comments will be provided in the discussion. 


\subsection{Test 2}

Test 2 investigates the influence of the car-following law at a platoon level. Now, the vehicle's trajectory is simulated using the simulated trajectory of its leader as an input. Thus, the traffic model is recursively applied from vehicle 3 to $n$ within a platoon and errors may propagate. This is confirmed by studying the mean RMSE, see Fig. 5. It increases from 0.5 to $0.6 \mathrm{~m} / \mathrm{s}$ for the Gipps model and from 0.7 to $1.1 \mathrm{~m} / \mathrm{s}$ for the Newell model. It seems that the Gipps model is less sensitive to the error propagation. One more time, from a traffic point of view the results globally remain good because the upper bound of the RMSE interval is $0.9 \mathrm{~m} / \mathrm{s}(2.1 \mathrm{~m} / \mathrm{s})$ for the Gipps (Newell) model.

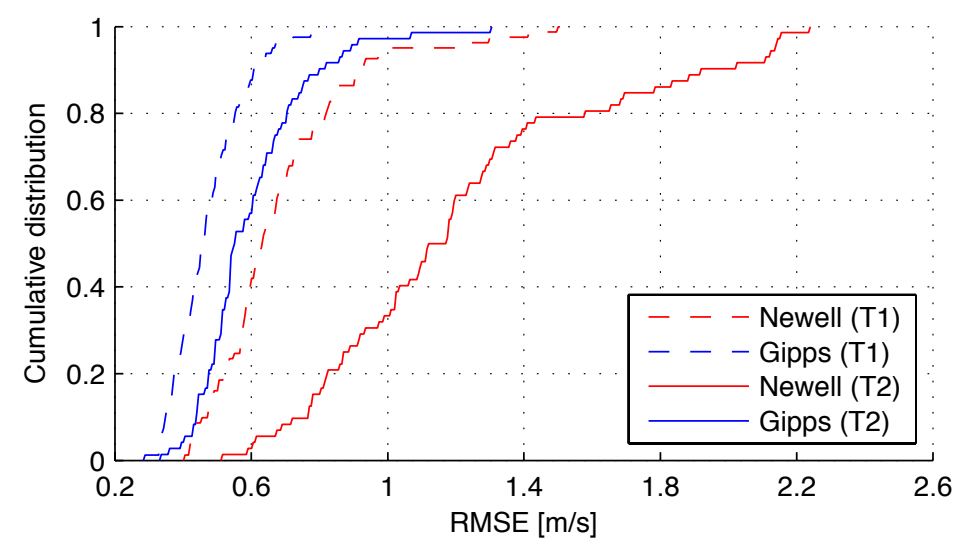

Fig. 5. RMSE cumulative distribution for all vehicle pairs - Test 2

Let us first analyze the fuel consumption outputs. The results for test 2 and $\varepsilon$ are very close to the results of test 1, see Fig. 6 - upper line and Tab. 5. Indeed, the mean $\varepsilon$ is equal to $-2.2 \%$ for the Newell model $(-16.9<\varepsilon$ $<11.2 \%)$ and to $-9 \%(-28.3<\varepsilon<4.2 \%)$ for the Gipps model. This means that the fuel consumptions are less sensitive than driving cycles to the propagation of errors related to the recursive application of the car-following model. This result is noticeable because test 2 is the common application case for traffic modeling. Fig. 6 also confirms that individual errors for fuel consumption may be very important (much more than for the RMSE) but this vanishes when the outputs are aggregated over all vehicles. This is confirmed by the analysis of the global error $E$ whose mean is equal to $-2.6 \%$ for Newell's model and to $-9.3 \%$ for Gipps' model. The discrepancies with test 1 and the same outputs are low. A very good point for the Newell model is that the length of the boundary interval $\left(5^{\text {th }}\right.$ and $95^{\text {th }}$ percentiles) for $E$ remains small, i.e. $10.3 \%$ compared to $5.9 \%$ for test 1 . This means that the compensation occurs for the Newell model even for a group of few vehicles (in the order of the size of a platoon, i.e. 10 vehicles). In comparison, the length of the same interval for Gipps' model and test 2 is equal to $25.8 \%$, which is a significantly worse score. 

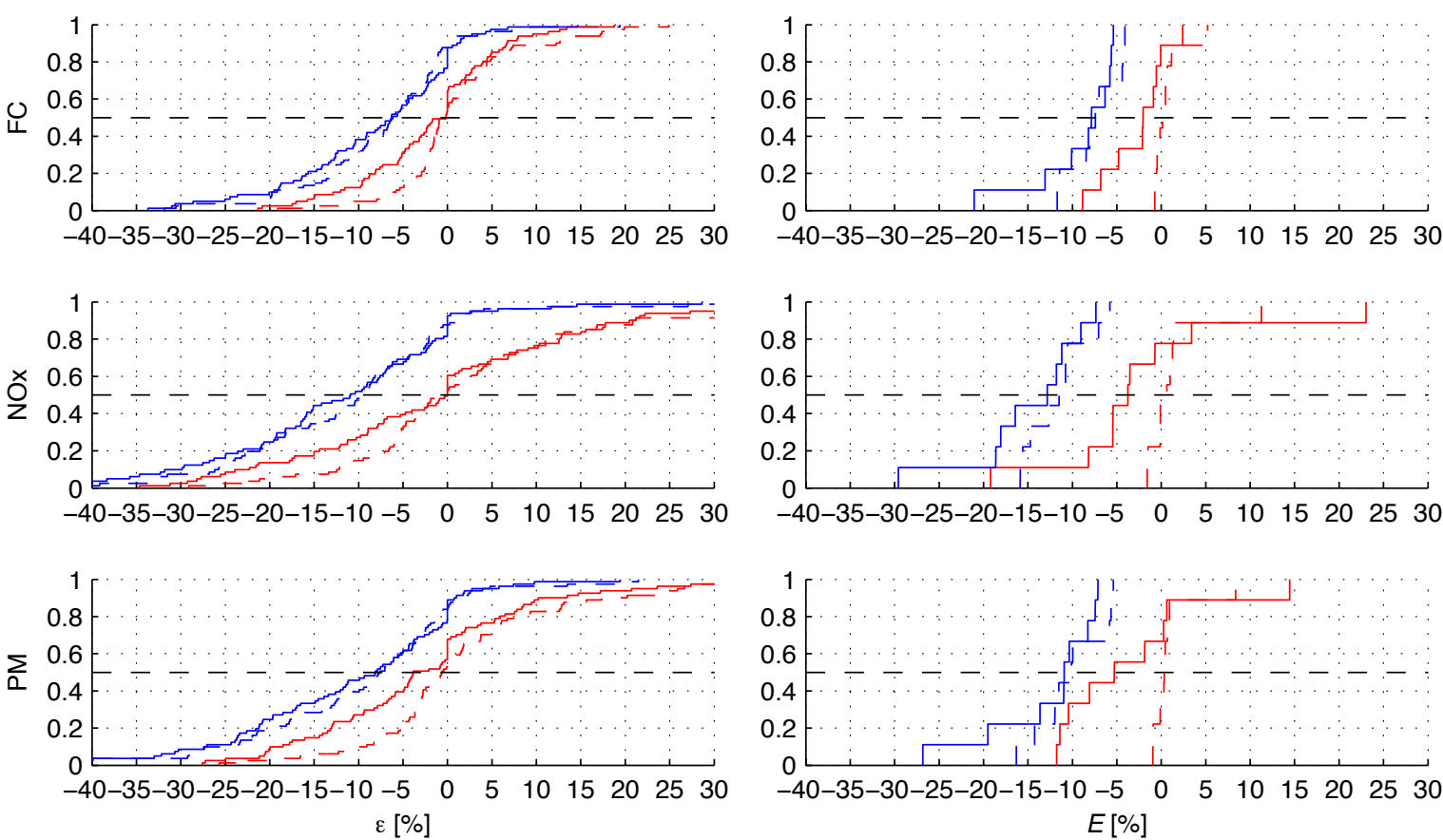

$---\operatorname{Newell~(T1)~-~-~-~Gipps~(T1)~Newell~(T2)~-~Gipps~(T2)~}$

Fig. 6. Cumulative distribution for the local and global errors - Test 2

The results for the other outputs (NOx and PM emissions) are provided in Fig. 6 and Tab. 5. They clearly confirm the previous conclusions, i.e. the Newell model provides accurate estimation at a platoon level while the Gipps model introduces more significant bias. Indeed, mean $E$ for the Newell (respectively Gipps) model is equal to $-2.2 \%(-14.1 \%)$ for NOx and $-2.8 \%(-12.8 \%)$ for PM. However, these two pollutants seem more sensitive to the errors generated by traffic models because the standard deviation values and the lengths of the boundary intervals either for $\varepsilon$ and $E$ are much higher than for the fuel consumption. This means that emission calculations should be aggregated over a higher number of vehicles than for fuel consumption to reach the same level of accuracy.

\begin{tabular}{cccccccccc}
\hline & & \multicolumn{3}{c}{ Newell's model } & \multicolumn{4}{c}{ Gipps's model } \\
& & $\boldsymbol{m}$ & $\boldsymbol{\sigma}$ & $\boldsymbol{q}_{\mathbf{5} \%}$ & $\boldsymbol{q}_{\mathbf{9 5} \%}$ & $\boldsymbol{m}$ & $\boldsymbol{\sigma}$ & $\boldsymbol{q}_{\mathbf{5} \%}$ & $\mathbf{q}_{\mathbf{9 5} \%}$ \\
\hline \multirow{4}{*}{ FC } & Real [g] & 31,5 & 6,8 & 23,9 & 43,1 & 34,9 & 7,7 & 26,2 & 47,5 \\
& Sim. [g] & 30,6 & 6,6 & 24,5 & 42,8 & 31,6 & 7,6 & 22,9 & 45,5 \\
& $\boldsymbol{\varepsilon}[\%]$ & $-2,2$ & 8,2 & $-16,9$ & 11,2 & $-9,0$ & 9,4 & $-28,3$ & 4,2 \\
& $E[\%]$ & $-2,6$ & 3,5 & $-8,9$ & 2,4 & $-9,3$ & 5,1 & $-21,1$ & $-5,4$ \\
\hline \multirow{4}{*}{ NOx } & Real [mg] & 261 & 70 & 170 & 380 & 286 & 76 & 191 & 414 \\
& Sim. [mg] & 255 & 68 & 169 & 381 & 244 & 72 & 165 & 382 \\
& $\boldsymbol{\varepsilon}[\%]$ & $-0,4$ & 18,6 & $-28,1$ & 37,1 & $-14,1$ & 13,5 & $-38,1$ & 5,4 \\
& $E[\%]$ & $-2,2$ & 11,3 & $-19,2$ & 23,0 & $-15,0$ & 6,7 & $-29,6$ & $-7,4$ \\
\hline
\end{tabular}




\begin{tabular}{cccccccccc}
\hline \multirow{4}{*}{ PM } & Real [mg] & 10,8 & 2,4 & 7,9 & 15,0 & 11,8 & 2,7 & 8,8 & 17,0 \\
& Sim. [mg] & 10,4 & 2,2 & 8,2 & 14,7 & 10,3 & 2,6 & 7,1 & 15,4 \\
& $\boldsymbol{\varepsilon}[\%]$ & $-2,8$ & 13,7 & $-21,1$ & 23,3 & $-12,3$ & 12,5 & $-32,9$ & 5,6 \\
& $E[\%]$ & $-3,7$ & 8,4 & $-11,8$ & 14,5 & $-12,8$ & 6,5 & $-26,8$ & $-7,1$ \\
\hline
\end{tabular}

Tab. 5: Emission results for Test 2

To conclude the analysis of test 2, Fig. 7 presents the evolution of the mean value $m_{k}$ of $\varepsilon$ over all the platoons for the same vehicle rank $k$. To highlight trends in the errors propagation, the absolute value of $m_{k}$ is plotted. The error increase between successive vehicles is clearly visible for the RMSE and the Newell model (see. Fig. 7a). Interestingly this is no longer the case for the Gipps model, which therefore proves to be significantly more robust from a traffic point of view. With regards to the different emission outputs, no clear tendency appears for the evolution of $m_{k}$ with respect to $k$. The evolution is slightly increasing for Gipps' model and slightly decreasing for Newell's model. The recursive application of the Newell model reduces the accuracy of the simulated trajectories but the associated driving cycles keep the key figures to provide accurate emission estimations. On the contrary, Gipps' model leads to better speed RMSE but this does not mean that the corresponding emissions are also well reproduced. A corollary is that the speed RMSE is not a good indicator to ensure that vehicle emission calculations would be accurate.

(a)

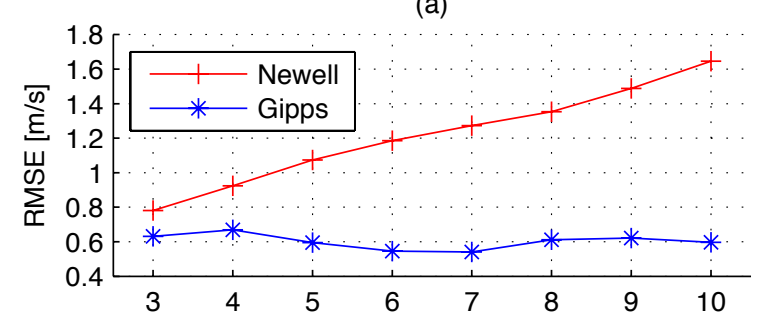

(c)

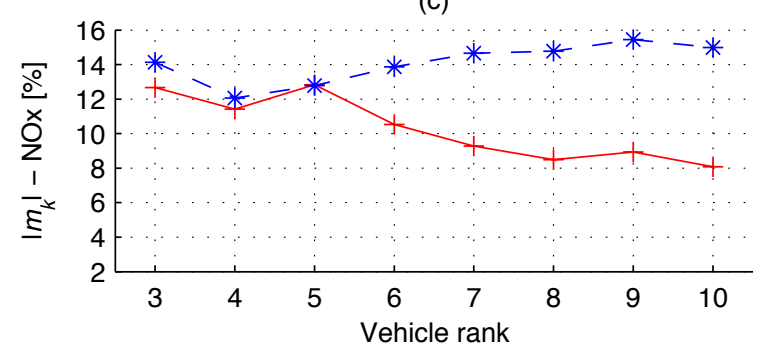

(b)

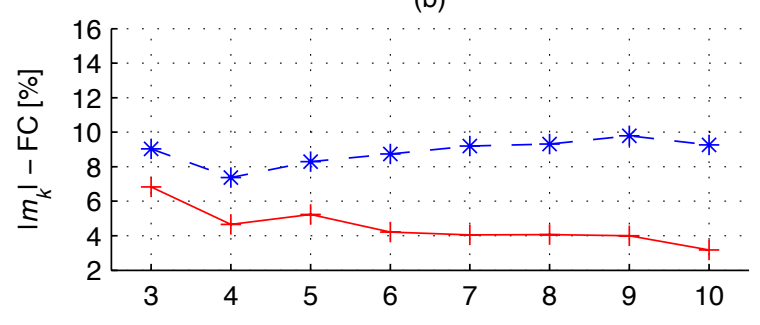

(d)

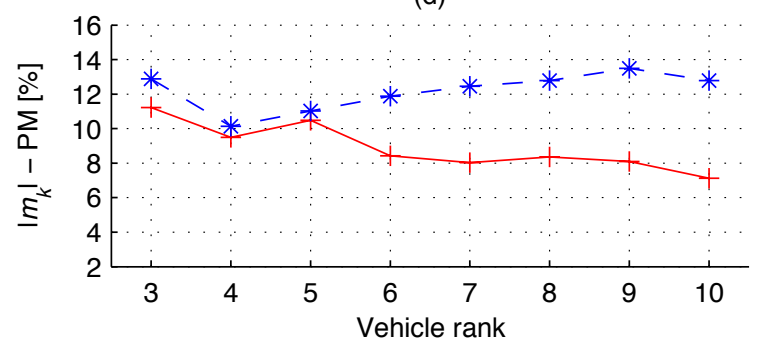

Fig. 7. Error propagation within platoons (a) Speed RMSE, (b) to (d) Mean local errors $\left|m_{k}\right|$ with respect to $k$

\subsection{Test 3}

Test 3 now investigates how non-optimal parameters influence the accuracy of fuel consumption and emission estimates. Parameter values are averaged over all vehicles. The resulting values are applied in both models as a single set of parameters for all individual vehicles. This corresponds to the classical case when traffic models are 
used without knowing the individual characteristic of each generated vehicle. The mean RMSE only slightly increase from 0.6 to $0.8 \mathrm{~m} / \mathrm{s}$ (respectively from 1.1 to $1.2 \mathrm{~m} / \mathrm{s}$ ) when comparing test 3 to test 2 for Gipps (respectively Newell). From a traffic point of view, this means that averaged parameters values provide a good approximation for the real behavior of individual vehicles. The upper bound of RMSE interval for Gipps is now $1.3 \mathrm{~m} / \mathrm{s}$ compared to $0.9 \mathrm{~m} / \mathrm{s}$ in test 2, see Fig. 8. The upper bound for Newell is unchanged. This confirms the relevance of using a single set of mean parameters for all platoons and all vehicles.

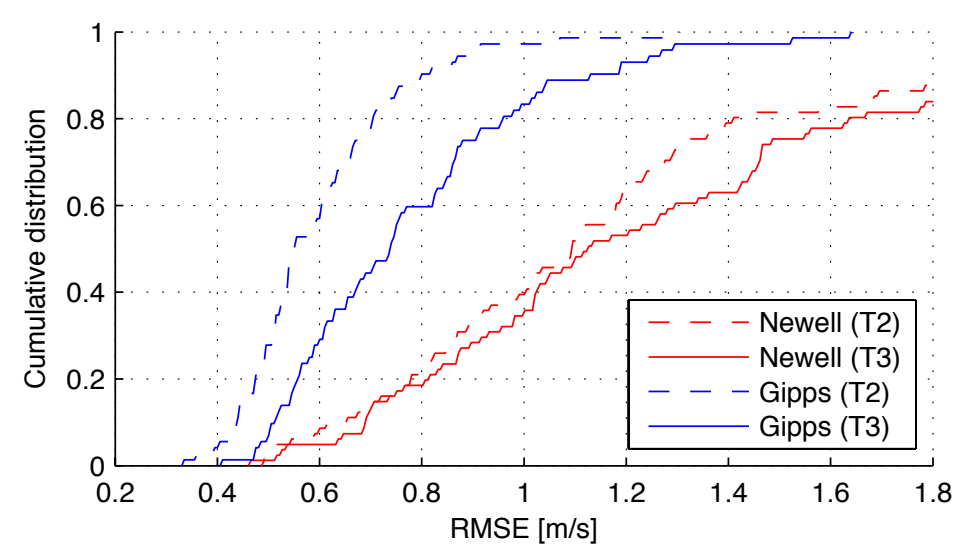

Fig. 8. RMSE cumulative distribution for all vehicle pairs - Test 3

The Newell model provides exactly the same emission outputs for tests 2 and 3, cf. section 3.1 for explanations. This is a very remarkable property of this model because using averaged instead of optimal parameter values only introduces discrepancies in the traffic behavior and not in the emission calculations. Indeed, the follower speed profile is not smoothed by the model and simply corresponds to the leader one. Newell's model is the only traffic model with this particularity. It is therefore well adapted for environmental applications.

Fig. 9 compares the cumulative distribution for tests 2 and 3 and the different emission outputs for the Gipps model. Surprisingly, the results are better for test 3 than for test 2 for all the outputs. This is confirmed when studying the different statistics provided in Tab. 6. For example, the mean $\varepsilon$ is equal to $-5.5 \%$ for fuel consumption, $-7.2 \%$ for NOx emissions and $-8.4 \%$ for PM emissions. The corresponding results for test 2 are $9 \%,-14.1 \%$ and $-12.3 \%$. The mean parameters appear more robust when emissions are calculated on average over all vehicles. Thus, mean but relevant traffic parameters seem sufficient to provide accurate emission estimates when all vehicles are considered. Again, this is not the case when vehicles are individually considered because the $\varepsilon$ intervals for this test are: from -24.7 to $-16.3 \%$ for fuel consumption, from $-32.9 \%$ to $-22 \%$ for NOx and from -38 to $-22.5 \%$ for PM. 
These conclusions are confirmed when studying the global error $E$ for all emission outputs, see Tab. 6. Mean $E$ values are in the same order as mean $\varepsilon$ values and are again more accurate for test 3 than for test 2 . It is worth noticing that the Gipps model always underestimates emissions either at a vehicle or a platoon level.
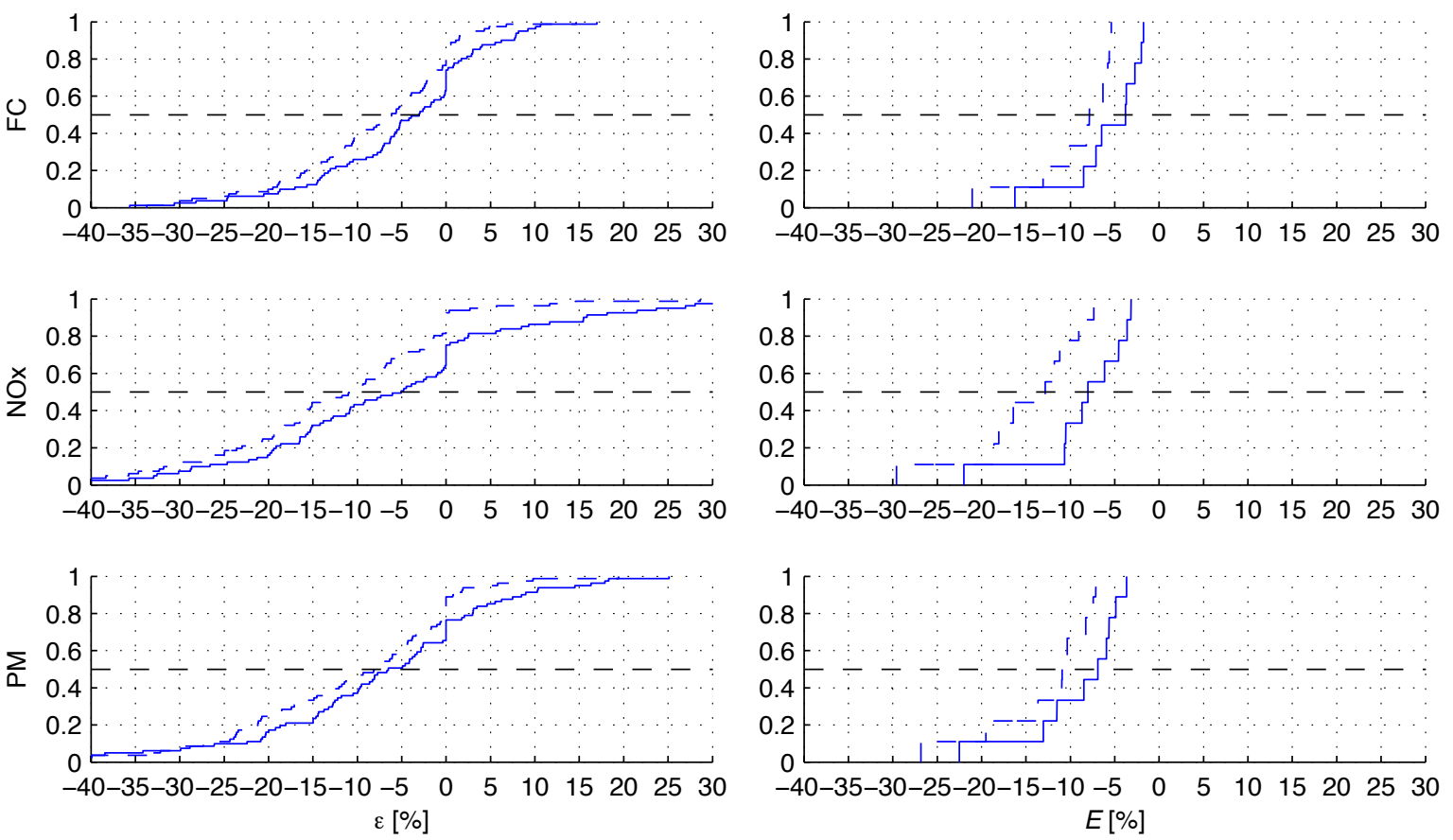

$$
-- \text { Gipps (T2) Gipps (T3) }
$$

Fig. 9. Cumulative distribution for the local and global errors - Test 3

\begin{tabular}{cccccc}
\hline & & \multicolumn{4}{c}{ Gipps's model } \\
& & $\boldsymbol{m}$ & $\boldsymbol{\sigma}$ & $\boldsymbol{q}_{\mathbf{5} \%}$ & $\boldsymbol{q}_{\mathbf{9 5} \%}$ \\
\hline \multirow{4}{*}{ FC } & Real [g] & 34,9 & 7,7 & 26,2 & 47,5 \\
& Sim. [g] & 32,8 & 7,5 & 24,8 & 47,1 \\
& $\boldsymbol{\varepsilon}[\%]$ & $-5,5$ & 10,2 & $-24,7$ & 9,1 \\
& $E[\%]$ & $-5,8$ & 4,6 & $-16,3$ & $-1,8$ \\
\hline \multirow{4}{*}{ NOx } & Real [g] & 286 & 76 & 191 & 414 \\
& Sim. [g] & 261 & 73 & 185 & 414 \\
& $\boldsymbol{\varepsilon}[\%]$ & $-7,2$ & 17,4 & $-32,9$ & 26,6 \\
& $E[\%]$ & $-8,6$ & 5,7 & $-22,0$ & $-3,2$ \\
\hline \multirow{4}{*}{ PM } & Real [g] & 11,8 & 2,7 & 8,8 & 17,0 \\
& Sim. [g] & 10,7 & 2,6 & 7,7 & 16,3 \\
& $\boldsymbol{\varepsilon}[\%]$ & $-8,4$ & 14,6 & $-38,0$ & 16,1 \\
& $E[\%]$ & $-9,2$ & 5,9 & $-22,5$ & $-3,7$ \\
\hline
\end{tabular}

Tab. 6: Emission results for Test 3 


\subsection{Global sensitivity analysis}

A global sensitivity analysis is now performed at the vehicle level focusing on $\varepsilon$ for the different emission outputs. Remember that the vehicle ID has been added as an input. Fig. 10a clearly shows that this last parameter overwhelms the others. Indeed, the associated $S_{i}$ is equal to 0.42 for FC, 0.49 for NOx and 0.49 for PM while the sum of all other $S_{i}$ only is respectively below $0.15,0.18$ and 0.14 see Fig. 10a2. This means that the calculation of a vehicle emissions is very sensitive to the use of the proper set of optimal parameters associated to this vehicle. It is not sufficient to apply a consistent set of parameters (in that case the optimal set of another vehicle). This result is quite trivial because it simply means that a careful calibration is required to obtain accurate results at a local (vehicle) level. What is interesting here is that we can quantify the need of calibration by looking at the relative importance of $S_{i}$. Note that this analysis has important practical applications. Indeed, the optimal parameters for a particular vehicle are not known but drawn within distributions that are not necessarily correlated. This confirms the results obtained in tests 1 to 3 : individual analyses for either fuel consumption or NOx and PM emissions are not relevant when a traffic model is used.

(a1)

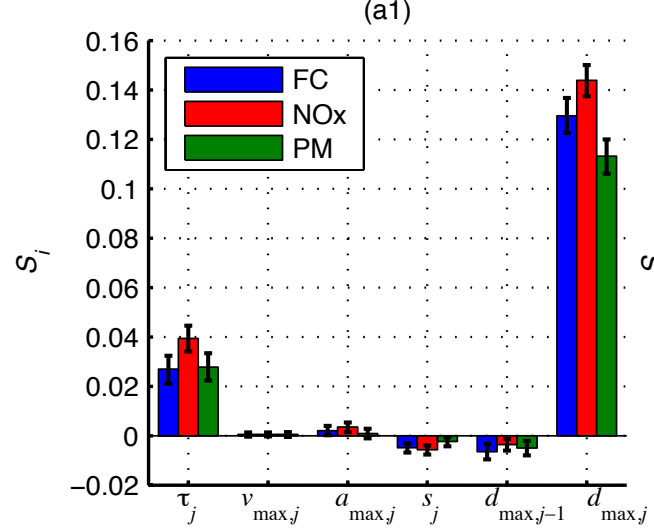

(b1)

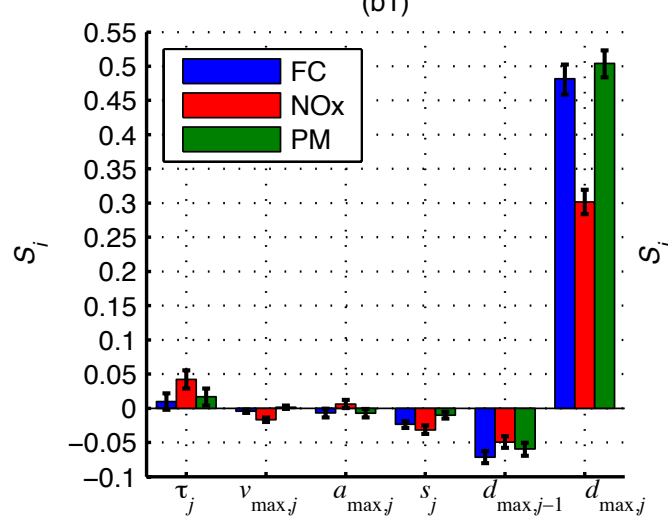

(a2)

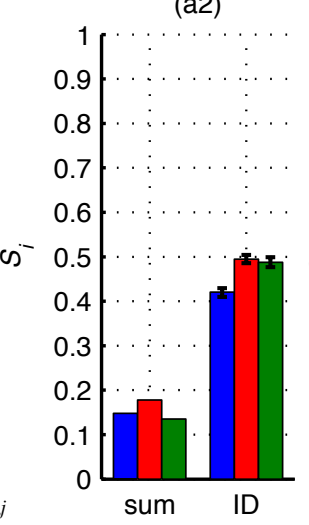

(b2)

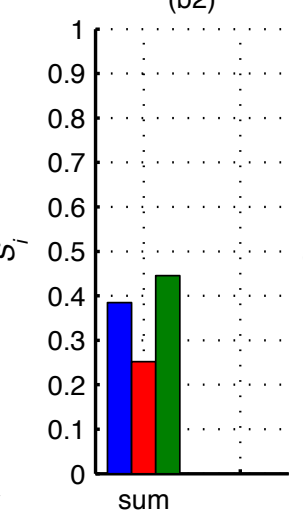

(a3)

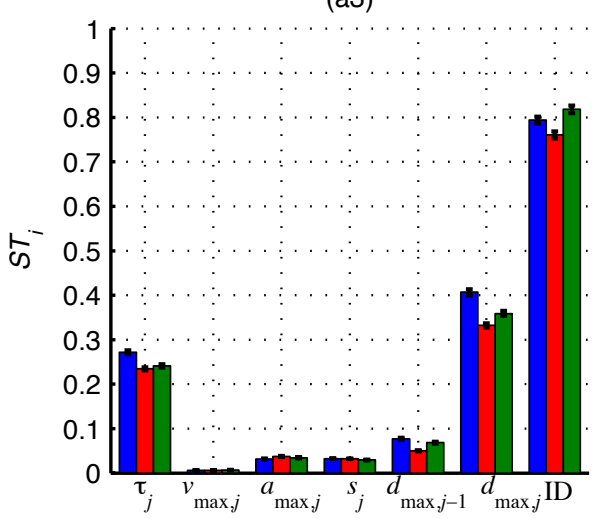

(b3)

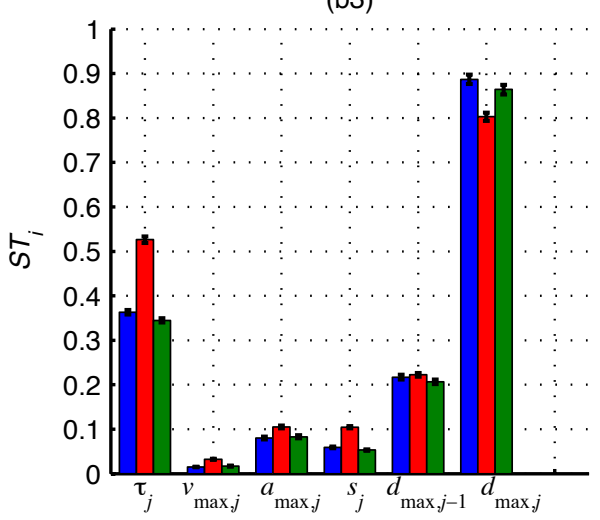

Fig. 10. Global sensitivity analysis (a) all vehicles (b) vehicle ID=2 (1) first order sensitivity index $S_{i}$ for all parameters except vehicle ID (2) $S_{i}$ for vehicle ID (3) Total effect index $S T_{i}$ 
When only analyzing the car-following parameters, it appears that the maximal deceleration of the follower $d_{\max , j}$ has the most influence on the emission calculations, see Fig. 10a1. Looking at the $S T_{i}$ values in Fig. 10a3 confirms this analysis. The reaction time $\tau_{j}$ seems also to be significant especially when considering its second order influence. Indeed, the $S T_{i}$ value for this last parameter is high and close to the $S T_{i}$ value of $d_{\max , j}$.

Finally, Fig. $10 \mathrm{~b}$ presents the results of the global sensitivity analysis for a particular vehicle ID=2. This allows testing the relative influence of the traffic parameters only for this particular case. This confirms that $d_{\max , j}$ and $\tau_{j}$ are the most influential parameters. They should be calibrated in priority and with the lower uncertainty bounds as possible.

\section{Discussion}

The first main conclusion of this paper is that a precise calibration of the car-following rules is not sufficient to get accurate estimates of the fuel consumption, the NOx and PM emissions at a vehicle level. Indeed, tests 1 and 2 show that the optimal parameters of both traffic models lead to highly distributed local errors (vehicle level) for all the emission outputs. A second result is that if Gipp's model appears more accurate that Newell's model from a traffic point of view this is no longer the case when the car-following rule is used to derive emission calculations. For such an application, the Newell model provides better results even if no model can be judged satisfactory when estimating the emission of individual vehicles if the calibration has been performed using goodness of fit indicator that are only related to the traffic behavior.

A solution is to introduce in the goodness of fit indicator criteria that account for the accuracy of emission calculations. This consists in switching to a multi-objective calibration method. For example, we can define an objective function $F$ that corresponds to a normalized mix between the speed RMSE and the local absolute error $|\varepsilon|$ associated to a given output (FC, NOx or PM):

$$
F(\rho)=(1-\rho) \frac{R M S E}{R M S E_{\max }}+\rho\left|\frac{\varepsilon}{\varepsilon_{\max }}\right|
$$

Fig. 11 presents the results of such a calibration method for the first three vehicles of the first platoon (ID=1 to 3 ) and the fuel consumption. Two $\rho$ values are tested: $\rho=0.5$ and $\rho=1$. Note that $\rho=0$ corresponds to the classical case studied in this paper. $\rho=1$ corresponds to a reverse situation where the accuracy from a traffic point of view is not considered. We use a classical global search algorithm provided by Matlab ${ }^{\mathrm{TM}}$ to find the optimum. Fig. 11a shows that when $\rho=0.5$, the fuel consumption error is drastically reduced while the speed RMSE only slightly increases. Indeed $\varepsilon$ is now always lower than $2 \%$ for the three considered vehicles while the maximal increase in the speed RMSE is $0.27 \mathrm{~m} / \mathrm{s}$. When $\rho=1$, the accuracy of the fuel consumption estimation is not really 
improved while the speed RMSE highly worsens. A calibration only based on fuel consumption is not valid for traffic applications and is not even necessary because it does not provide significantly better results. Fig. 11b highlights these conclusions by comparing the calibrated trajectories for vehicle ID $=2$ and $\rho=0,0.5$ and 1 .

This preliminary result is really appealing because it tends to demonstrate that a multi-objective calibration method can well address the joined problem of accurate traffic and emission representations. It has to be confirmed with the extensive analysis of all vehicles and all emission outputs. This has not been realized yet because introducing the emission model in the optimization loop makes the calculation very slow (more than 2 days for a single full run). The authors are currently investigating a solution that approximates the emission model by a Krigging meta-model (Kleijnen, 2009) to drastically speed up the calculations.
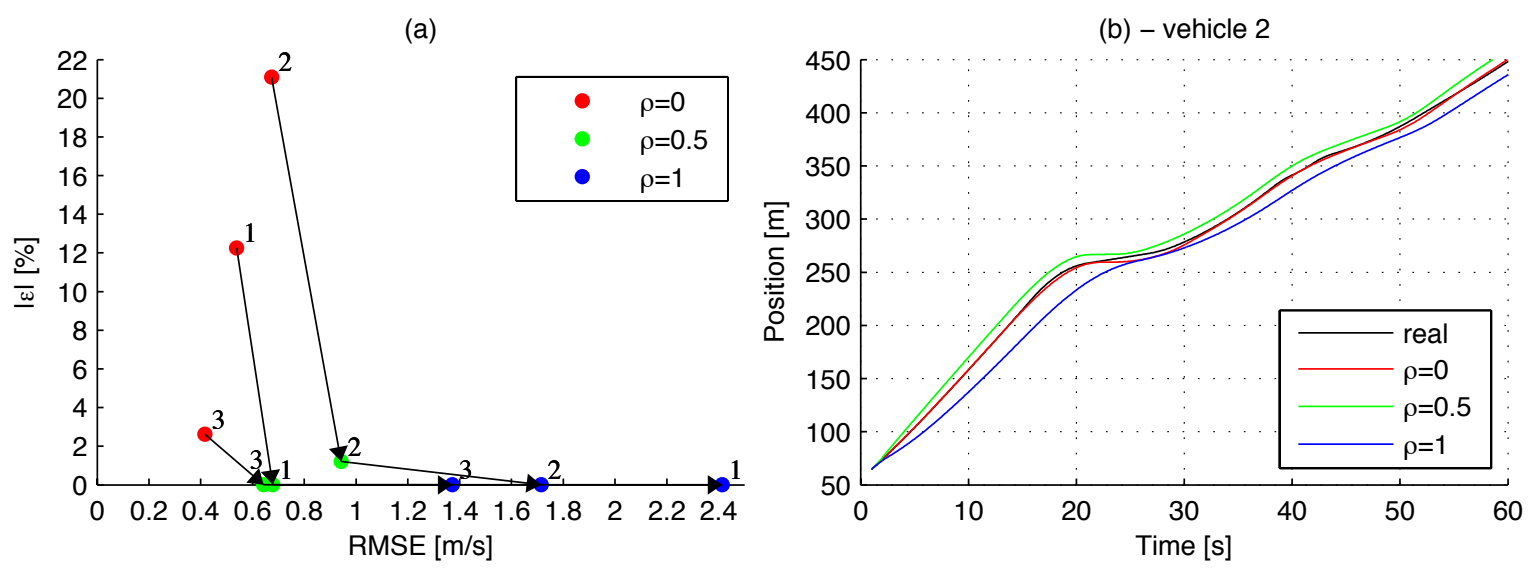

Fig. 11. Multi-objective calibration results for three particular vehicles (ID=1 to 3 )

The second main conclusion of this paper is that optimal or even averaged traffic parameters seem sufficient to estimate the fuel consumption and the NOx and PM emissions for a group with many vehicles (global or traffic level). Thus, if the emissions can hardly be estimated for a particular vehicle using model parameters only calibrated for traffic purpose, this is no longer the case for platoons or for the total traffic. Indeed, local errors on a driving cycle that have significant impact for the vehicle emissions at a local and individual scale compensate when lots of driving cycles are considered. This confirms the conclusions from a previous study that only focused on fuel consumption (Vieira da Rocha et al, 2013). This is particularly relevant as in the practice different strategies and investments on a transportation system are evaluated at the aggregated level rather than at the level of the single vehicle.

\section{References}

Brockfeld, E., Kühne, R., Wagner, P., 2004. Calibration and validation of microscopic traffic flow models. Transportation Research Record, 1876:62-70.

CETU, 2012 Calcul des émissions de polluants des véhicules automobiles en tunnel. $<$ www.cetu.developpement-durable.gouv.fr/IMG/pdf/CETU_DocInfo_Calcul_des_Emissions_2012.pdf>. (accessed 11.12.13). 
Chiabaut, N., Leclercq, L., Buisson, C., 2010. From heterogeneous drivers to macroscopic patterns in congestion. Transportation Research Part B, 44(2):299-308.

Chiabaut, N., Buisson, C., Leclercq, L., 2009. Fundamental diagram Estimation through passing rate measurements in congestion. IEEE transactions on Intelligent Transportation Systems, 10(2):355-359.

Ciuffo, B., Punzo, V., Torrieri, V., 2008.A comparison between simulation-based and model-based calibrations of traffic flow micro-simulation models. Transportation Research Record, 2088:36 - 44.

Ciuffo, B., Punzo, V., Montanino, M., 2012. 30 years of the Gipps' car following model: applications, developments and new features. Transportation Research Record, 3350:1-19.

Federal Highway Administration, 2006. NGSIM - Next Generation SIMulation. <www.fhwa.dot.gov/publications/research/operations/06137/index.cfm/>. (accessed 31.10.08).

Gipps, P.G., 1981. A behavioural car-following model for computer simulation. Transportation Research Part $B, 15(2): 105-111$.

Hausberger, S., Rexeis, M., Zallinger, M., Luz, R., 2009. Emission factors from the model PHEM for the HBEFA version 3. TUG, Report I-20/2009, 1-76.

Int Panis, L., Broekx, S., Liu, R., 2006. Modelling instantaneous traffic emission and the influence of traffic speed limits. Science of the Total Environment, $371: 270-285$.

Kleijnen, J.P.C., 2009. Kriging metamodeling in simulation. European Journal of Operational Research, $192: 707-716$.

Lighthill, M.J., \& Whitham, G.B., 1955. On kinematic waves: II. A theory of traffic flow on long crowded roads. Proceedings of the Royal Society, 229(1178):317-345.

Leclercq, L., Chiabaut, N., Laval, J., Buisson, C., 2007. Relaxation phenomenon after changing lanes: an experimental validation with the NGSIM dataset. Transportation Research Record, 1999:79-85.

Luz, R., Hausberger, S., Rexeis, M., 2013. The Emission Model PHEM. TUG Technical report, 2p. http://www.ivt.tugraz.at/de/forschung/emissionen.html. (accessed 14.02.2014).

Marczak, F., Buission, C., 2012. A new filtering method for trajectories measurement errors and its comparison with existing method. Transportation Research Record, 2315:35-46.

Newell, G.F., 2002. A simplified car-following theory: a lower order model. Transportation Research Part B, 36(3):195-205

Ossen, S., Hoogendoorn, S.P., 2008. Validity of Trajectory-Based Calibration Approach of Car-Following Models in Presence of Measurement Errors. Transportation Research Record, 2088:117-125.

Punzo, V., Ciuffo, B., 2011. Sensitivity analysis of car-following models: methodology and application. In: Transportation Research Board 90th Annual Meeting - Paper \#11-2805, 18p.

Punzo, V., Simonelli, F., 2005. Analysis and Comparison of Microscopic Traffic Flow Models with Real Traffic Microscopic Data. Transportation Research Record, 1934:53-63.

Richards, P.J., 1956. Shock waves on the highway. Operations Research. 4:42-51.

Saltelli, A., Ratto, M., Andres, T., Campolongo, F., Cariboni, J., Gatelli, D. Saisana, M., Tarantola, S., 2008. Global sensitivity analysis, The Primer, Wiley.

Sobol, I.M., 1990. On sensitivity estimation for nonlinear mathematical models. Matem. Mod., 2(1):112-118.

Toledo, T., Ben-Akiva M. E., Darda D., Jha M., Koutsopoulos, H., 2004. Calibration of Microscopic Traffic Simulation Models with Aggregate Data. Transportation Research Record, 1876:10-19.

Toledo, T., Koutsopoulos, H. N., \& Ben-Akiva, M., 2009. Estimation of an integrated driving behavior model. Transportation Research Part C, 17(4):365-380.

Treiber, N., Kesting, A., Thiemann, C., 2008. How much does traffic congestion increase fuel consumption and emissions? Applying a Fuel Consumption Model the NGSIM Trajectory Data. In: Transportation Research Board 87th Annual Meeting, Paper \#08-2715, 17p.

Trigui, R., Jeanneret, B., \& Badin, F., 2004. Systemic modelling of hybrid vehicles in order to predict dynamic performance and energy consumption. Recherche Transports Sécurité, 83 :147-150.

Vieira da Rocha, T., Can, A., Parzani, C., Jeanneret, B., Trigui, R., Leclercq, L., 2013. Evaluating the influence of microscopic traffic models outputs on fuel consumption estimation. Transportation Research part D, 24:1726.

Wilson, R.E., 2001. An Analysis of Gipps' Car-Following Model of Highway Traffic. IMA Journal of Applied Mathematics, 66(5):509-537. 